'Departamento de Neurología y Neurocirugía, Hospital Clínico de

la Universidad de Chile. Facultad de Medicina. Universidad de Chile.

${ }^{2}$ Unidad de Neurocirugía, Clínica Alemana de Santiago.

${ }^{a}$ Alumno $7^{\circ}$ Año de Medicina Facultad de Medicina Universidad de Chile

Recibido el 20 de agosto de 2013, aceptado el 7 de agosto de 2014.

Correspondencia a: Alfredo Yáñez L. Santos Dumont $9992^{\circ} \mathrm{E}$ Independencia, Santiago. Teléfono: 27773882 alermanda@vtr.net

\section{Tratamiento de la hemorragia subaracnoidea aneurismática en el Hospital Clínico de la Universidad de Chile}

\author{
ALFREDO YÁÑEZ LERMANDA ${ }^{1,2}$, ARTURO RUIZ-ABURTO AGUILAR ${ }^{a}$
}

Background: One third of patients with subarachnoid hemorrhage caused by intracranial aneurysms, die. Aim: Review of medical records of patients with subarachnoid hemorrhage treated at a clinical hospital. Material and Methods: Review of medical records of patients discharged from the hospital between 2006 and 2011 with the diagnosis of subarachnoid hemorrhage. Patients initially or subsequently treated elsewhere were not analyzed. Results: The medical records of 82 patients aged 24 to 100 years (77\% females), were analyzed. The clinical diagnosis at the onset of the condition was correct in $82 \%$ of cases. In $95 \%$ of patients, an angiographic study and subsequent surgical intervention of the aneurysm were carried out within 24 hours of diagnosis. Global mortality was $23 \%$. Twelve patients died prior to any possible treatment, due to the severity of the disease. Seventy six aneurysms in 70 patients were treated with clips or coils in 37 and 39 cases, respectively. Seven patients died. Forty seven patients had a Rankin disability score of two or less. Conclusions: The presence of an intracranial hematoma or acute hydrocephaly on admission and clinical vasospasm during evolution were associated with a bad prognosis.

(Rev Med Chile 2014; 142: 982-988)

Key words: Intracranial aneurysms; Subarachnoid haemorrhage; Treatment.

L a hemorragia subaracnoidea (HSA) por rotura de un aneurisma es una enfermedad grave. Un tercio de los pacientes fallecen las horas siguientes a causa de la hemorragia y los dos tercios restantes enfrentan una situación de riesgo vital derivada de múltiples factores que pueden complicar el cuadro inicial en los días o semanas siguientes. El manejo oportuno, anticipando y corrigiendo los factores de riesgo para estas complicaciones marca una diferencia significativa en el desenlace final.

La HSA aneurismática tiene una incidencia en Chile de 6,2 casos x 100.000 habitantes ${ }^{1}$ algo me- nor que la estimada desde estudios poblacionales extranjeros ${ }^{2,3}$.

Las complicaciones más frecuentes y graves de la HSA aneurismática son el resangrado, que suele producirse dentro de la primera semana, la hidrocefalia aguda por obstrucción de las vías de pasaje del líquido cerebroespinal y el vasoespasmo, relacionado con la presencia de sangre en las cisternas subaracnoideas y que puede conducir secundariamente al desarrollo de áreas de infarto cerebral.

El riesgo de complicaciones graves está en directa relación con la latencia en el diagnóstico 
y el tratamiento. En nuestro país, desde el año 2007, la HSA causada por un aneurisma cerebral es una de las patologías protegidas por la Ley No 19.966 de Garantías explícitas en Salud (GES) la cual obliga a confirmar la sospecha clínica de HSA dentro de $24 \mathrm{~h}$, a una confirmación diagnóstica del aneurisma roto por angiografía TAC multicorte o angiografía digital dentro de las $48 \mathrm{~h}$ siguientes, $\mathrm{y}$ al tratamiento definitivo en las $24 \mathrm{~h}$ posteriores a la confirmación del diagnóstico ${ }^{4}$.

La eliminación del aneurisma se ha realizado durante muchos años mediante el clipaje quirúrgico directo ${ }^{5-8}$. En los últimos 15-20 años, la embolización con coils, a través de navegación endovascular, ha demostrado ser también una alternativa eficaz y válida ${ }^{9}$. Sigue abierto algún debate acerca de la elección del tratamiento más conveniente para cada tipo de aneurisma ${ }^{10-12}$.

El objetivo de este estudio es describir la experiencia del manejo de un grupo sucesivo de pacientes atendidos en el Hospital Clínico de la Universidad de Chile (HCUCh) con el diagnóstico comprobado de HSA de origen aneurismático, con el fin de analizar sus características demográficas, la precisión del diagnóstico en la primera consulta, el momento de la atención respecto del protocolo GES, además del tipo de tratamiento realizado, comparando la evolución y desenlace de acuerdo a las opciones de tratamiento elegidas. Se comparan dichos resultados confrontándolos con la literatura internacional pertinente.

\section{Materiales y Métodos}

Se realizó un estudio descriptivo de los antecedentes clínicos de pacientes diagnosticados y tratados por HSA de causa aneurismática en el Servicio de Neurología y Neurocirugía del HCUCh. La selección de pacientes se realizó en base a la información histórica de los egresos hospitalarios, seleccionando a todos los pacientes que tuvieron el diagnóstico de HSA espontánea de causa aneurismática entre el mes de enero de 2006 y el mes de diciembre de 2011. Fueron excluidos de la revisión las fichas de aquellos pacientes con diagnóstico de HSA aneurismática que ingresaron para continuar un tratamiento comenzado en otro establecimiento y los pacientes intervenidos en nuestro hospital que fueron trasladados posteriormente a otros centros.
De un total de 105 pacientes con el diagnóstico de HSA aneurismática se descartan 16 pacientes trasladados precozmente a otros centros y 7 pacientes que ingresaron para continuación de manejo iniciado en otro lugar, se analizan finalmente las fichas de 82 pacientes.

Por ser un análisis retrospectivo, no es posible obtener el dato de aquellos pacientes en que se sospechó una HSA aneurismática con un estudio posterior negativo.

Para el análisis de datos se utilizó el programa Stata $10 \mathrm{SE}$, realizando pruebas de asociación estadística univariada con $\chi^{2}$.

\section{Resultados}

De los 82 pacientes que ingresaron al estudio, 63 fueron mujeres $(76,83 \%)$. El promedio de edad fue de 53,1 años (SD 12,24, rango 24-100 años).

Ante la sospecha clínica de una HSA se solicitó una tomografía computarizada cerebral (TC) sin contraste como método de confirmación diagnóstica. Sesenta y siete pacientes $(81,71 \%)$ fueron diagnosticados correctamente desde un comienzo como un cuadro de HSA espontánea. Ocho pacientes fueron catalogados como "accidente cerebrovascular" 4 pacientes como "migraña".

La confirmación por TC se logró dentro de las primeras $24 \mathrm{~h}$ de comenzado los síntomas en $93,9 \%$ de los pacientes (77) y el $100 \%$ a las $48 \mathrm{~h}$.

La Tabla 1 detalla la gravedad en la escala de la WFNS (World Federation of Neurosurgical Societies $)^{13}$ y la magnitud de la HSA según la escala de Fisher ${ }^{14}$.

Luego de la confirmación diagnóstica todos los pacientes fueron ingresados a la Unidad

Tabla 1. Puntaje WFNS y Fisher al ingreso

\begin{tabular}{|cccccr|}
\hline WFNS & $\begin{array}{c}\mathbf{n} \\
\text { pacientes }\end{array}$ & $\%$ & Fisher & $\begin{array}{c}\mathbf{n} \\
\text { pacientes }\end{array}$ & $\%$ \\
1 & 34 & 41,5 & 1 & 3 & 3,7 \\
2 & 11 & 13,4 & 2 & 14 & 17,1 \\
3 & 13 & 15,9 & 3 & 18 & 21,9 \\
4 & 6 & 7,3 & 4 & 47 & 57,3 \\
5 & 18 & 21,9 & & & \\
Total & 82 & 100,0 & Total & 82 & 100,0 \\
\hline
\end{tabular}


Hemorragia subaracnoidea por aneurisma intracraneal - A. Yáñez Lermanda et al

de Cuidados Intensivos a cargo de un equipo multidisciplinario (intensivista, neurocirujano, neurólogo vascular).

Se solicitó el estudio angiográfico inmediatamente posterior a la estabilización inicial del paciente. Este examen se realizó en 95,12\% (78 pacientes) dentro de las $24 \mathrm{~h}$ siguientes de la confirmación tomográfica de HSA.

El tipo de aneurisma diagnosticado y la modalidad de tratamiento se muestran en la Tabla 2; 12 pacientes no fueron intervenidos debido a un rápido deterioro o por la gravedad al ingreso. Se encontró más de un aneurisma en 6 casos por lo que la cantidad total de aneurismas asciende a 88 .

Una vez identificado el aneurisma cada paciente fue analizado en conjunto por un neurocirujano y un neuroradiólogo intervencional con el fin de decidir la modalidad de exclusión del aneurisma. Se intervinieron 70 pacientes $(85,36 \%), 65$ de los cuales fueron intervenidos excluyendo el aneurisma dentro de las $24 \mathrm{~h}$ siguientes al estudio angiográfico. Los pacientes que fueron tratados después de las $24 \mathrm{~h}$ corresponden a inconvenientes de logística (cantidad adecuada de clips o coils, pabellón adecuado disponible) en 4 casos. Un paciente que se consideró inicialmente para tratamiento conservador finalmente fue tratado tardíamente con coils.

El promedio de días de hospitalización fue de 29,75 días (SD 34,97, rango 1-180 días). Las hospitalizaciones cortas correspondieron a pacientes graves que fallecieron en las primeras horas o días.

El 69,51\% (57 pacientes) presentó algún tipo de complicación neurológica, siendo la más frecuente el vasoespasmo en $48,78 \%$ y la hidrocefalia en 22 pacientes $(26,83 \%), 18$ de estos últimos requirieron un drenaje ventricular externo previo al clipaje o coiling. Ambas situaciones se correlacionaron significativamente con la cantidad de sangre presente en el TAC inicial (Tabla 3 ).

Se observó algún tipo de complicación extraneurológica en $63,41 \%$ (52 pacientes), siendo las más frecuentes los trastornos de la deglución y las infecciones pulmonares. No hubo diferencias estadísticamente significativas del número de complicaciones en relación a la modalidad de intervención realizada.

El estado al alta de los pacientes, medida por la escala de Rankin ${ }^{15,16}$ en relación con su estado al ingreso se detalla en la Tabla 4. Fallecieron 7 pacientes, la mayoría de ellos ingresados en
Tabla 2. Tipos de Aneurismas y modalidad de exclusión recibida

\begin{tabular}{|c|c|c|c|c|c|}
\hline \multirow[t]{2}{*}{$\begin{array}{l}\text { Tipo de } \\
\text { aneurisma }\end{array}$} & \multicolumn{4}{|c|}{$\begin{array}{c}\text { Tipo de } \\
\text { tratamiento }\end{array}$} & \multirow[t]{2}{*}{$\begin{array}{c}p \\
\text { value }\end{array}$} \\
\hline & Total & $\begin{array}{c}\text { No } \\
\text { tratados }\end{array}$ & Clip & Coil & \\
\hline AcoA & 26 & 2 & 7 & 17 & $<0,05$ \\
\hline AcoP & 12 & 1 & 2 & 9 & NS \\
\hline ACM & 31 & 5 & 22 & 4 & $<0,05$ \\
\hline $\mathrm{ACl}$ & 7 & 1 & 3 & 3 & NS \\
\hline AOft & 2 & 0 & 1 & 1 & NS \\
\hline$A B$ & 3 & 2 & 0 & 1 & NS \\
\hline AV & 1 & 0 & 0 & 1 & NS \\
\hline PICA & 1 & 1 & 0 & 0 & NS \\
\hline ACA & 5 & 0 & 2 & 3 & NS \\
\hline $\begin{array}{l}\text { Total } \\
\text { aneurismas }\end{array}$ & 88 & 12 & 37 & 39 & NS \\
\hline
\end{tabular}

AcoA: Arteria comunicante anterior. AcoP: Arteria comunicante posterior. ACM: Arteria cerebral media. ACl: Arteria carótida interna. AOft: Arteria oftálmica. AB: Arteria basilar. AV: Arteria vertebral. PICA: Arteria cerebelos aposterior inferior. ACA: Arteria cerebral anterior.

Tabla 3. Porcentaje de presencia de vasoespasmo e hidrocefalia aguda en relación al grado de Fisher al ingreso

\begin{tabular}{|lcccc|}
\hline & \% & \multicolumn{2}{c}{ Fisher } & p value \\
& & $\mathbf{1 - 2}$ & $\mathbf{3 - 4}$ & \\
Vasoespasmo & 48,8 & 23,5 & 55,4 & $<0,05$ \\
Hidrocefalia aguda & 26,8 & 5,8 & 32,3 & $<0,05$ \\
\hline
\end{tabular}

Tabla 4. Puntaje de Rankin al alta según grado de WFNS preoperatorio

\begin{tabular}{|ccccccc|}
\hline $\begin{array}{c}\text { Rankin } \\
\text { al alta }\end{array}$ & $\mathbf{1}$ & $\mathbf{2}$ & $\mathbf{3}$ & $\mathbf{4}$ & $\mathbf{5}$ & Total \\
0 & 9 & 0 & 0 & 0 & 0 & 9 \\
1 & 24 & 4 & 4 & 0 & 0 & 32 \\
2 & 2 & 2 & 2 & 1 & 0 & 7 \\
3 & 2 & 1 & 1 & 0 & 0 & 4 \\
4 & 0 & 1 & 1 & 0 & 0 & 2 \\
5 & 2 & 0 & 0 & 5 & 2 & 9 \\
6 & 0 & 0 & 0 & 0 & 7 & 7 \\
Total & 39 & 8 & 8 & 6 & 9 & 70 \\
\hline
\end{tabular}


Tabla 5. Causa de fallecimiento según tratamiento

\begin{tabular}{|lcccc|}
\hline Causa & $\begin{array}{c}\text { Tipo de } \\
\text { tratamiento } \\
\text { Coil }\end{array}$ & $\begin{array}{c}\text { No } \\
\text { Clip }\end{array}$ & Total \\
HIC (hematoma/ & 1 & 2 & 8 & 11 \\
hemoventrículo) & 1 & 1 & 1 & 3 \\
Vasoespasmo/Infarto & 1 & 0 & 3 & 3 \\
Falla orgánica múltiple & 0 & 0 & 0 & 2 \\
Neumonía & 1 & 1 & 12 & 19 \\
\hline Total & 3 & 4 & & \\
\hline
\end{tabular}

Tabla 6. Fallecidos por rango de edad

\begin{tabular}{|ccc|}
\hline Edad & Total & Fallecidos \\
\hline $21-30$ & 4 & 1 \\
$31-40$ & 18 & 3 \\
$41-50$ & 17 & 1 \\
\hline $51-60$ & 17 & 5 \\
$61-70$ & 11 & 3 \\
\hline $71-80$ & 11 & 3 \\
\hline 80 o más & 4 & 3 \\
\hline Total & 82 & 19 \\
\hline
\end{tabular}

Tabla 7. Relación entre presencia de hidrocefalia aguda, vasoespasmo y hematoma intracerebral respecto del puntaje de Rankin al alta

\begin{tabular}{|lcccc|}
\hline & n/total & Rankin $\mathbf{4}$ o +/Total & Pearson $\boldsymbol{\chi}^{\mathbf{2}}$ & Valor $\mathbf{p}$ \\
Hidrocefalia & $22 / 82$ & $15 / 22$ & 12,9379 & $<0,01$ \\
Vasoespasmo & $40 / 82$ & $10 / 40$ & 4,5180 & 0,034 \\
Hematoma IC & $12 / 82$ & $10 / 12$ & 13,2411 & $<0,01$ \\
\hline
\end{tabular}

malas condiciones neurológicas. Treinta y nueve pacientes pudieron reincorporarse a sus labores previas. Las causas de fallecimiento y la edad de los fallecidos se muestran en las Tablas 5 y 6 siendo la presencia de un hematoma intracerebral la causa más frecuente de deterioro, de la misma forma que la edad avanzada. No hubo diferencias significativas en el desenlace relacionadas con la modalidad de exclusión del aneurisma.

La presencia de hidrocefalia aguda, de un hematoma intracerebral y vasoespasmo, se correlacionaron significativamente con peores resultados al alta (Tabla 7).

\section{Discusión}

La forma clínica más frecuente de presentación de un aneurisma cerebral es una HSA. Este suele ser un fenómeno de inicio brusco y violento con una cefalea ictal "la peor de mi vida", y que puede ir acompañado por distintos grados de compromiso de conciencia o focalidad. Se trata de un cuadro grave, que conlleva una mortalidad inmediata (antes de llegar a un centro asistencial) de alrede- dor de $10 \%$. Frente a la sospecha clínica es posible diagnosticarla fácilmente en las horas iniciales mediante imágenes de TC sin contraste, que revelará la presencia de sangre en el espacio subaracnoideo. La sensibilidad del examen decrece en el tiempo a medida que la sangre se reabsorbe o recircula. El diagnóstico oportuno de esta condición tiene una importancia crucial, ya que el mayor riesgo de una resangrado está en los 2 ó 3 primeros días de evolución. La sospecha clínica de HSA se basa casi exclusivamente en datos anamnésticos y con cierta frecuencia puede ser confundida con cefaleas de otra índole (migraña, cefalea de tensión) situación que retrasa el tratamiento. En esta serie el diagnóstico de HSA fue planteado desde un comienzo en $82 \%$. El advenimiento de la TC como un examen de relativo fácil acceso facilitó el diagnóstico precoz, aun en aquellos casos en que la sospecha clínica fue diferente. La legislación chilena privilegia la atención de estos pacientes ofreciendo garantías de oportunidad acordes a la letalidad de la enfermedad, que está relacionada a la hemorragia en sí misma o a complicaciones que pueden desarrollarse desde las primeras horas de desencadenado el episodio. De los pacientes que se 
Hemorragia subaracnoidea por aneurisma intracraneal - A. Yáñez Lermanda et al

hospitalizan, algo menos de un tercio fallece antes de lograr una estabilización eficiente.

Posterior a la confirmación diagnóstica de la HSA, una angiografía o angiotomografía mostrará en la gran mayoría de los casos la presencia del aneurisma causante. El algoritmo de atención GES establece un plazo de $48 \mathrm{~h}$ luego de confirmada la HSA, para realizar un estudio angiográfico y $24 \mathrm{~h}$ más para la exclusión del aneurisma.

Las complicaciones precoces más graves de esta patología son la ocurrencia de una nueva hemorragia o la obstrucción aguda de las vías de circulación del líquido cerebroespinal (LCE) produciendo una hidrocefalia aguda. Más tardíamente pueden aparecer vasoespasmo o alteraciones crónicas de reabsorción del LCE. El riesgo de vasoespasmo (y eventuales infartos secundarios) y de hidrocefalia están directamente relacionados a la magnitud de la hemorragia inicial.

El primer plan terapéutico frente a una HSA aneurismática una vez estabilizado el paciente es, por consecuencia, la exclusión del aneurisma causante y las alternativas válidas de tratamiento son el clipaje directo mediante una craneotomía o la embolización con coils.

El tratamiento de un aneurisma roto es multifactorial y por lo tanto multidisciplinario. Implica, por una parte, eliminar precozmente la causa de la hemorragia (exclusión del aneurisma) y por otro lado anticipar, prevenir y corregir fenómenos asociados a la presencia de sangre en el espacio subaracnoideo, principalmente vasoespasmo y/o hidrocefalia. La mantención del equilibrio del medio interno, de la hemodinamia y de la función pulmonar juegan un rol crucial en el desenlace. El detalle del manejo por parte del intensivista en estos pacientes está fuera de los objetivos de este trabajo.

La estabilización general del paciente incluyó una derivación ventricular externa en los casos de hidrocefalia aguda con deterioro del puntaje de Glasgow (18 pacientes). El estudio angiográfico se hizo en forma precoz ( $95 \%$ dentro de las primeras $24 \mathrm{~h}$ siguientes al diagnóstico) concordante con lo establecido en el protocolo de atención de la Ley GES. En esta serie no se intervinieron pacientes con imágenes de angiotomografía (AngioTAC). La exclusión del aneurisma se planificó para completarse dentro de las $24 \mathrm{~h}$ posterior a la angiografía, según la modalidad acordada entre el neurocirujano y el neuroradiólogo, idealmente a continuación al procedimiento diagnóstico. En los casos derivados a cirugía diagnosticados durante la tarde/noche, se prefirió diferir el procedimiento por algunas horas hasta la mañana siguiente con el fin de contar con un pabellón y equipo tratante sin las limitaciones propias de un pabellón de urgencia con personal menos habituado a las complejidades de este tipo de cirugía.

Si bien la modalidad de tratamiento usado para exclusión del aneurisma ha sido controversial durante algún tiempo, es actualmente aceptado que ambas técnicas (clip o coil) son válidas y complementarias, la infraestructura y experiencia del equipo humano de cada centro determina la elección final de la técnica a utilizar la mayor parte de las veces ${ }^{17,18,19}$.

En nuestra serie hemos privilegiado el tratamiento mediante acceso quirúrgico directo en aquellos pacientes portadores de un hematoma expansivo, los aneurismas de bifurcación de cerebral media y los distales de cerebral anterior (pericallosos). Los aneurismas de circulación posterior, del complejo de comunicante anterior o carotideos proximales se consideró en primera instancia el tratamiento endovascular. Sobre estos lineamientos generales los matices y variantes anatómicas fueron analizados en conjunto con el fin de decidir la mejor opción de tratamiento (Por Ej. En un aneurisma en el origen de la comunicante posterior con polígono tipo fetal se prefiere el tratamiento quirúrgico).

Los pacientes WFNS 4 y 5 fueron derivados a embolización, a excepción de aquellos casos en que esta condición era causada por hipertensión intracraneana secundaria a un hematoma intracraneano los que fueron sometidos al vaciamiento del hematoma y clipaje.

Hubo 12 pacientes que fallecieron dentro de la primera semana a pesar de las medidas de soporte iniciales sin ser sometidos a procedimientos de exclusión del aneurisma.

Existió una relación pronóstica estadísticamente significativa de mayor riesgo (puntaje de Rankin 4 o más) respecto a la presencia de una hidrocefalia aguda o a un hematoma intracerebral al comienzo de la enfermedad, la misma tendencia se observa en aquellos pacientes que desarrollaron un vasoespasmo durante su evolución, aunque no alcanzaron una diferencia estadísticamente significativa en el análisis univariado.

Como está demostrado de estudios anterio- 
Hemorragia subaracnoidea por aneurisma intracraneal - A. Yáñez Lermanda et al

res $^{20,21}$ el vasoespasmo ocurrió significativamente con mayor frecuencia en pacientes portadores de HSA extensas (puntajes de Fisher 3 o más) lo mismo que la ocurrencia de hidrocefalia que fue más frecuente en aquellos pacientes que tenían mayor cantidad de sangre en las cisternas basales a su ingreso.

La letalidad global de la HSA en esta serie (23\%) es comparable a lo referido en otros estudios extranjeros ${ }^{2,22}$. La mortalidad entre los 70 pacientes a los cuales fue posible tratar el aneurisma fue de $10 \%$ (7/70) sin existir diferencias estadísticamente significativas respecto de la modalidad de tratamiento del aneurisma. Los días de permanencia en la UTI y de hospitalización total tampoco muestran diferencias entre ambas poblaciones, lo que es reflejo del tratamiento global en la unidad de cuidados intensivos posterior a la exclusión del aneurisma el que fue similar para ambos grupos.

Existió una correlación importante entre el puntaje WFNS de ingreso y el desenlace, habiendo mejores resultados en aquellos pacientes que ingresaron en mejores condiciones. La excepción la constituyen 5 pacientes con un grado 5 WFNS al ingreso secundario a la existencia de una hidrocefalia aguda o a un hematoma intraparenquimatoso que finalmente lograron un Rankin de 2 o menor. En la contraparte, existen 5 pacientes con puntajes WFNS de 1 ó 2 al ingreso, tres de los cuales fallecieron ( 2 por vasoespasmo e infartos y 1 por neumonia tardía) y otros 2 con un puntaje de Rankin al alta de 5, como consecuencia de infartos extensos secundarios a vasoespasmo ${ }^{23}$.

Existen consenso que los resultados del tratamiento de aneurismas rotos están relacionados al volumen de pacientes atendidos; los centros en los cuales se concentra este tipo de patología suelen tener mejores estadísticas ${ }^{24,25,26}$. Los resultados obtenidos en nuestra institución, a pesar de tener un volumen relativamente bajo de casos tratados, son comparables e incluso algo superiores al promedio reportado en la literatura, lo que atribuimos al trabajo en equipo, a la selección cuidadosa de la mejor modalidad de exclusión del aneurisma para cada caso en particular y a que los procedimientos fueron realizados siempre por parte de o con la colaboración directa de los especialistas de mayor experiencia en patología vascular.

Existen muy escasos reportes de la experiencia nacional en el tratamiento de la HSA aneurismática $^{27}$ debiendo extrapolar desde la literatura extranjera las indicaciones y modalidades de tratamiento adecuándolas a nuestra realidad. Hemos considerado valioso la publicación de nuestros resultados con el fin de dar a conocer la experiencia dentro de nuestro medio en el tratamiento de una patología altamente demandante de recursos de infraestructura y de capital humano.

El uso adecuado de la indicación de exclusión de un aneurisma roto debiera atravesar por la consideración de ambas técnicas ${ }^{27}$. Es preciso recalcar que la toma de decisiones respecto de la modalidad de exclusión de un aneurisma roto, en aquellos centros en los cuales se cuenta con ambos recursos, debiera ser hecha por un equipo vascular completo, es decir con la presencia de ambos especialistas responsables del procedimiento: neurocirujanos y neuroradiólogos intervencionales y no plantear, ni al paciente ni a su familia, opiniones previas acerca de la modalidad de tratamiento antes de la revisión de todas las imágenes y los antecedentes completos del caso por parte de un equipo multidisciplinario.

\section{Referencias}

1. Lavados PM, Sacks C, Prina L. Incidence, 30-day Casefatality Rate and Prognosis of stroke in Iquique, Chile: a 2-year Community-based Prospective Study (PISCIS proyect). Lancet 2005; 365 (9478): 2206-15.

2. Muller TB, Sandvei MS, Kvistad KA. Unruptured Intracranial aneurysms in the Norwegian HUNT-syudy: Risk of Rupture Calculated from Data in a Population-based Cohort Study. Neurosurgery 2013 Apr.25 [Epub ahead of print].

3. Rincón F, Rossenwasser RH, Dumont A. The Epidemiology of Admissions of Non-traumatic Subarachnoid Hemorrhage in the United States. Neurosurgery 2013; 73 (2): 217-22.

4. República de Chile, Ministerio de Salud 2007. Guía Clínica Hemorragia Subaracnoidea Secundaria a Rotura de Aneurismas Cerebrales. Disponible en www.minsal. cl [Consultado el 20 de marzo de 2012].

5. Dandy WE. Intracranial aneurysm of internal carotid artery cured by operation. Ann Surg 1938; 107: 654.

6. Norlén G, Olivecrona $H$. The treatment of aneurysms of the circle of Willis. J Neurosurg 1953; 10: 404-15.

7. Kurze T. Microtechniques in neurological surgery. Clin Neurosurg 1964; 11: 128-37.

8. Yasargil MG, Vise WM, Bader DC. Technical adjuncts in neurosurgery. Surg Neurol 1977; 8: 331-6.

9. Guglielmi G, Viñuela F, Sepetka I. Electrothrombosis 
Hemorragia subaracnoidea por aneurisma intracraneal - A. Yáñez Lermanda et al

of saccular aneurysms via endovascular approach. Part 1: electrochemical basis, technique, and experimental results. J Neurosury 1991; 75: 1-7.

10. Molineux AJ, Kerr RSC, Stratton I. International Subarachnoid Aneurysm Trial (ISAT) of neurosurgical clipping versus endovascular coiling ln 2143 patients with ruptured intracranial aneurysms: a randomised trial. Lancet 2005; 366 (9488): 809-17.

11. Tait MJ, Critchley GR, Norris JS. How much can be concluded from the international Subarachnoid Aneurysm Trial (ISAT)? Brit. J. Neurosurg 2007; 21 (1): 3-6.

12. Mitchell P, Kerr R, Mendelow D, Molineux A. Could late rebleeding overturn the superiority of cranial aneurysm coil embolization over clip ligation seen in the International Subarachnoid Aneurysm Trial? J Neurosurg 2008; 108: 437-42.

13. Teasdale GM, Drake CG, Hunt W, Kassell N, Sano K, Pertuiset B. A Universal Subarachnoid Hemorrhage Scale: Report of a Committee of the World Federation of Neurosurgical Societies. J Neurol Neurosurg Psychiatry 1988; 51: 1457.

14. Fisher CM, Kistler JP, Dais JM. Relation of cerebral Vasospasm to Subarachnoid hemorrhage visualized by Computarized Tomographic Scanning. Neurosurgery 1980; 6 (1): 1-9.

15. Quinn TJ, Dawson J, Waters MR, Lees KR: Reliability of the modified Rankin Scale: A Systematic Review. Stroke 2009; 40 (10): 3393-5.

16. Keun-Sik Hong, Saver JL. Quantifying the Value of Stroke Disability Outcomes: WHO Global Burden od Disease Proyect Disability Weights for Each Level of Modified Rankin Scale. Stroke 2009; 40 (12): 3828-33.

17. Lanzino G, Fraser K, Kanaanm, Wagenbach A. Treatment of ruptured intracranial aneurysms since the International Subarachnoid Aneurysm Trial: Practice utilizing clip ligation and coil embolization as individual or complementary therapies. J. Neurosurg 2006; 104: 344-9.

18. Burrows AM, Korumilli R, Lanzino G. How we do it:
Acute management of subarachnoid hemorrhage. Neurol Res 2013; 35 (2): 111-6.

19. Guerrero F, De La Linde C, Pino FI. Manejo general en Cuidados intensivos del paciente con hemorragia subaracnoidea espontánea. Med. Intensiva 2008; 32 (7): 342-53.

20. Claassen J, Bernardini G, Kreiter K. Effect of Cisternal and Ventricular Blood on Risk of Delayed Cerebral Ischemia After Subarachnoid Hemorrhage The Fisher Scale Revisited. Stroke 2001; 32: 2012-20.

21. Frontera J, Claassen J, Schmidt JM. Prediction of Symptomatic Vasospasm After Subarachnoid Hemorrhage: The Modified Fisher Scale. Neurosurgery 2006; 59: 21-7.

22. Ronne-Engström E, Enblad P, Gál G, Norbäck O, Ryttlefors M, Cesarini KG. Patients with spontaneous subarachnoid haemorrhage-presentation of a 10-year hospital series. Br J Neurosurg 2009; 23 (5): 499-506.

23. Wang T, Zhang JH, Qin X. Analysis on death-associated factors of patients with subarachnoid hemorrhage during hospitalization. Acta Neurochir Suppl 2011; 110 (Pt 1): 219-23.

24. Mc Neill L, English SW, Borg N. Effects of Institutional Case Load of Subarachnoid hemorrhage on mortality: A Secondary Analysis of Administrative Data. Stroke 2013; (3) 647-52.

25. Natarajan SK, Sekhar LN, Ghodke B, Britz GW. Outcomes of ruptured intracranial aneurysms treated by microsurgical clipping and endovascular coiling in a high-volume center. AJNR Am J Neuroradiol 2008; 29 (4): 753-9.

26. Cross DT 3rd, Tirschwell DL, Clark MA. Mortality Rates after Subarachnoid Hemorrhage: Variation According to Hospital Case Volume in 18 States. J Neurosurg 2003; 99 (5): 810-7.

27. Torrealba G, Lorenzoni J, Tagle P, et al. Resultado de la Cirugía en Aneurismas Intracraneanos rotos y Análisis de las Causas de Morbimortalidad. Neurocirugía/Neurocirurgía 1996; 7 (1): 42-8. 